\title{
Deep Vein Thrombosis Simulating Hamstring Injury on Sonography
}

The hamstrings are some of the most frequently injured muscles, commonly associated with exercise and athletic activity. ${ }^{1,2}$ Although muscle injury can often be diagnosed clinically, imaging evaluation is important to help determine the severity, guide treatment, and monitor healing. Sonography is increasingly being used to evaluate tendons and muscle injuries and has many advantages over other imaging modalities. In some cases, deep vein thrombosis (DVT) may mimic tendon or muscle injury. ${ }^{2,3}$ Deep vein thrombosis has a high incidence among the general population, and even with adequate therapy it can in some cases evolve to pulmonary embolism and death. ${ }^{4}$ Sonography can thus be a useful modality for evaluating musculoskeletal pain and separating muscle or tendon injury from DVT.

A 36-year-old male patient with a medical history notable for Crohn disease presented at the family medicine department with left hamstring pain. Approximately 7 days earlier, he reported some soreness and cramping in his left hamstring after he was mountain climbing. He denied any specific injury but stated that the activity was a moderate amount of exercise for him. Three days precise to presenting, he was playing basketball and again felt some tightness in his left hamstring area. As self-treatment, he took ibuprofen, wrapped his thigh, and applied ice to the affected area, with some pain relief. He went to see his family physician after worsening of the thigh pain.

Physical examination revealed some swelling in the left hamstring compared to the right, but there was no obvious ecchymosis, erythema, or fluid collection. He appeared to have full range of motion of the hips bilaterally. His gait was within normal limits but slightly favored the left side. He had some tenderness in the left hamstring area when asked for flexion of his knee joint. He had no tachycardia or shortness of breath. The patient did not mention a previous episode of superficial thrombosis in his distal right lower extremity and was not asked specifically about a history of venous thrombosis.

The physician clinically diagnosed left hamstring strain. He advised local ice, ibuprofen, light stretching, and a slow and gradual return to activity and ordered lower extremity sonography to confirm his diagnosis. Two days later, the patient underwent a sonographic evaluation of his left hamstring injury. There was no sonographic evidence of injury to the hamstring origin or muscle complex. Diffuse edema was noted throughout the posterior thigh to the knee with muscle and subcutaneous skin thickening (Figure 1A). Given the normal hamstring and tendon and 
the unexplained leg swelling, the sonographer continued to examination the thigh to the level of the knee and noted that the femoral vein just proximal to the knee joint appeared prominent with internal echoes and decreased compressibility (Figure 1B). A dedicated vascular study was performed, which confirmed DVT occlusion of the femoral, popliteal, peroneal, and profunda femoris veins. The age of the thrombus was indeterminate. ${ }^{5}$

The patient was immediately sent to the emergency department. His pulse was 110 to 115 beats per minute; his blood pressure was 123/74 mm Hg; and his oxygen saturation was $99 \%$ on room air. Because of his tachycardia, a computed tomographic pulmonary angiogram was performed and showed a left lower lobe segmental and subsegmental embolism (Figure 1C). The patient received anticoagulation and has done well over the past 7 months.

Sonography has been shown to accurately detect a variety of muscle and tendon injuries. Hamstring injuries are commonly associated with exercise, affecting not only professionals but also amateur athletes. Although muscle in-

Figure 1. Deep vein thrombosis simulating hamstring injury in a 36-year-old man. A, Sonography of the posterior aspect of the bilateral thigh. Left, The left thigh shows diffuse swelling, increased echogenicity consistent with edema, and marked skin thickening. Right, The right thigh is normal on sonography. B, Longitudinal sonography of the femoral vein shows increased internal echogenicity due to a thrombus. C. Axial postcontrast computed tomography shows segmental left lower lobe pulmonary emboli. Additional subsegmental emboli were also present (not shown).

A

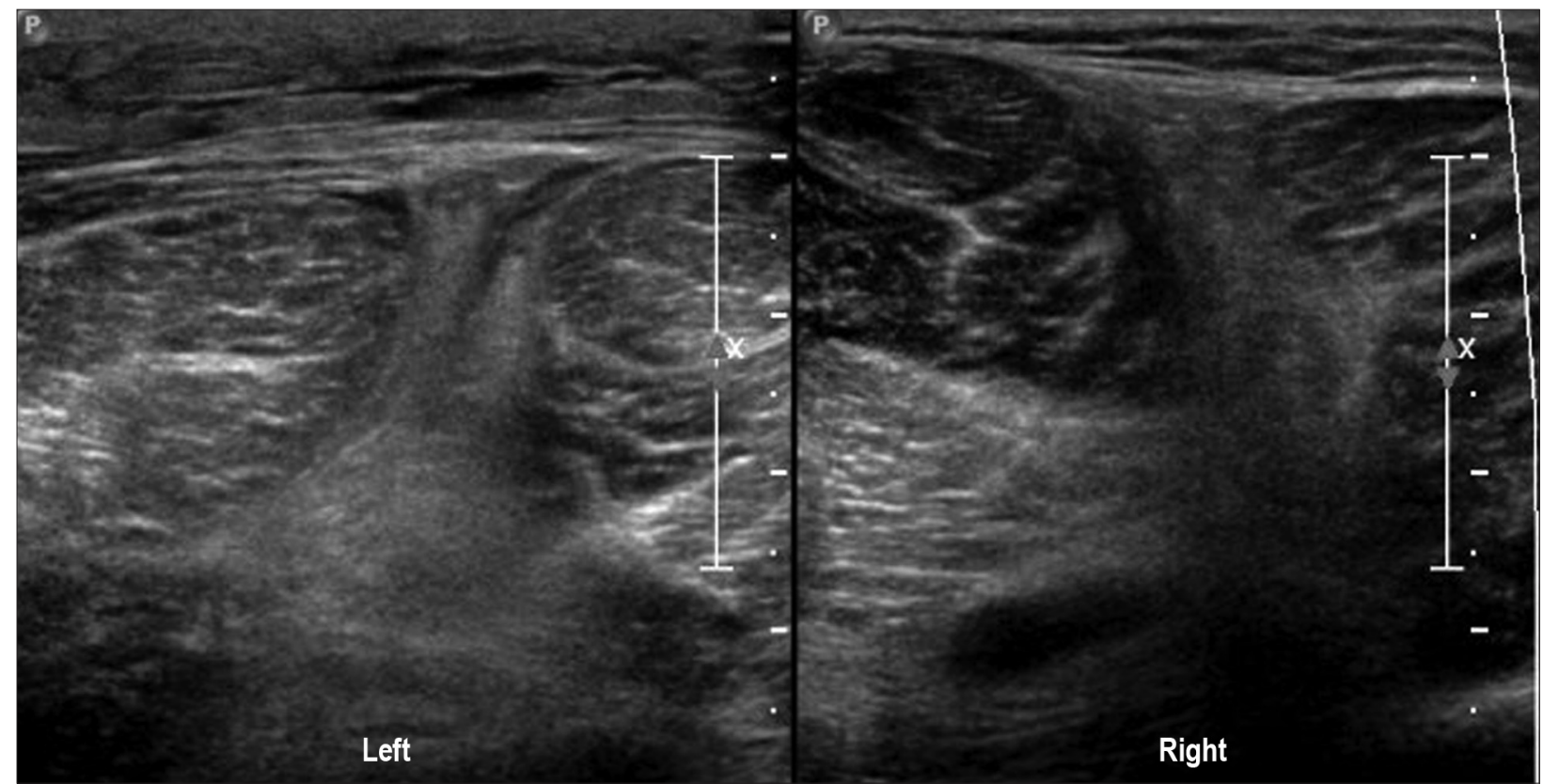

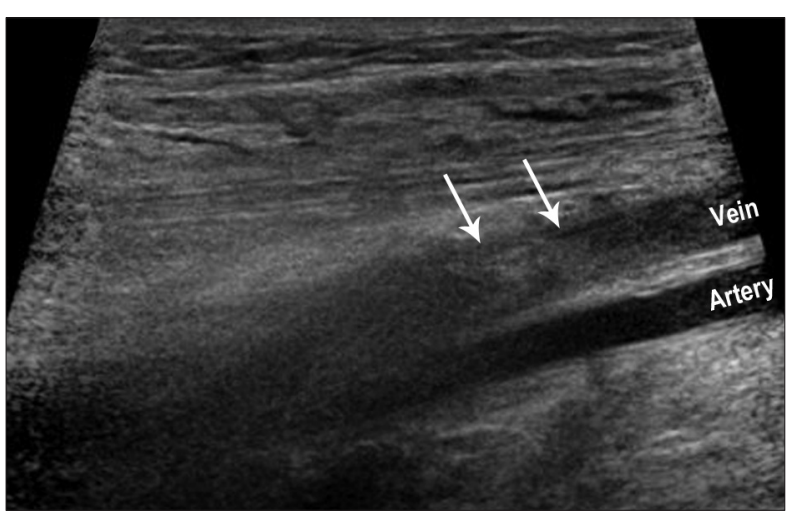

C

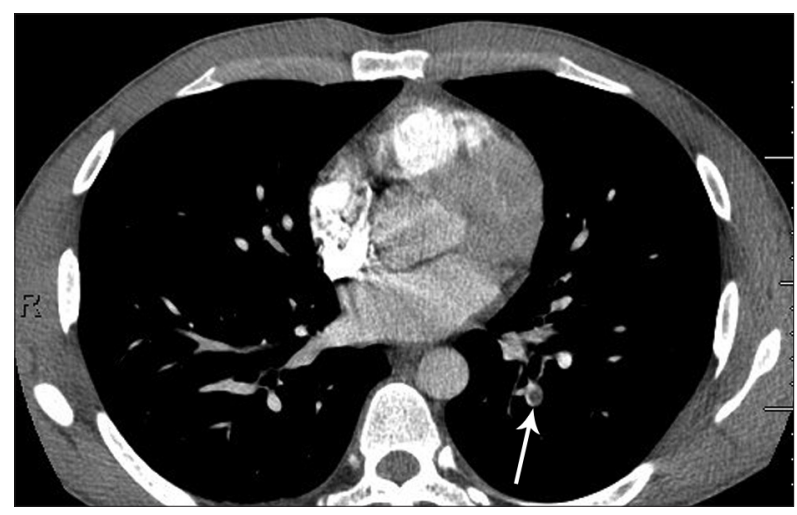


juries can often be clinically diagnosed, imaging is a useful way to diagnose injury, monitor healing, and exclude other causes, including DVT, which can in some cases mimic a muscle tear. ${ }^{2,3}$

Because of its high accuracy, sonography is a commonly used modality for evaluation of DVT. ${ }^{3,4,6}$ Compression sonography for lower limbs has sensitivity of $95 \%$ and specificity of $98 \%$ for symptomatic proximal DVT and sensitivity and specificity of $11 \%$ to $100 \%$ and $90 \%$ to $100 \%$, respectively, for symptomatic distal DVT. According to a standard diagnostic algorithm, sonography is indicated when there is a low pretest probability of DVT but a positive $\mathrm{D}$-dimer test result and always when there is a high pretest that probability of DVT even if the D-dimer test result is negative. 3

Sonography is increasingly being used for evaluation of tendon and muscle injuries. It has no patient contraindications, allows a more comfortable examination, and avoids a prolonged and potentially uncomfortable examination that can occur with magnetic resonance imaging. ${ }^{7}$ Sonography has a higher resolution than magnetic resonance imaging, which aids evaluation of tendon tears and other superficial structures. ${ }^{7}$ Some musculoskeletal pathologic conditions and functional information, such as the function of tendons and ligaments, are not present when patient is at rest. Sonography allows a real-time joint, ligament, muscle, or tendon dynamic examination of the patient. ${ }^{7}$ It also provides a direct correlation with the site of the pain, increasing the chance of detecting the abnormality, and offers real-time clinical correlation. ${ }^{7}$

As shown in this case, Doppler sonography gives important physiologic information about blood flow, aiding diagnosis of DVT and helping differentiate cystic from solid masses. ${ }^{7}$ Sonography has an ease of contralateral comparison examination, detecting bilateral asymmetry that can be useful in deciding whether an abnormality is present. ${ }^{7}$

Sonography also has a flexible field of view. It allows the sonographer wide latitude in moving from one anatomic region to another. This facility is important for larger or longer structures in the body ${ }^{7}$ and was crucial for this case, in which the hamstring was normal, but diffuse leg edema was noted. In this case, the sonographer was able to quickly and easily move from the hamstring to the popliteal fossa and thus found the thrombi in the femoral vein. In addition, the flexible field of the view of sonography, wisely applied by the sonographer, turned a case of suspected hamstring strain into a diagnosis of DVT and ultimately pulmonary embolism and may have saved the patient's life.
Ricardo A. Lutterbach-Penna, MD, Monica Kalume-Brigido, MD, Brian L. Robertson, AS, RDMS, Jon A. Jacobson, MD, Gandikota Girish, MD, David P. Fessell, MD

Division of Musculoskeletal Radiology University of Michigan Health System

Ann Arbor, Michigan USA

\section{References}

1. Koulouris G, Connell D. Hamstring muscle complex: an imaging review. Radiographics 2005; 25:571-586.

2. Counsel P, Breidahl W. Muscle injuries of the lower leg. Semin Musculoskelet Radiol 2010; 14:162-175.

3. Tan M, van Rooden CJ, Westerbeek RE, Huisman MV. Diagnostic management of clinically suspected acute deep vein thrombosis. Br J Haematol 2009; 146:347-360.

4. Scarvelis D, Wells PS. Diagnosis and treatment of deep-vein thrombosis. CMAJ2006; 175:1087-1092.

5. Hamper UM, DeJong RM, Scoutt LM. Ultrasound evaluation of the lower extremity veins. Radiol Clin North Am 2007; 45:525-547.

6. Ho WK. Deep vein thrombosis: risks and diagnosis. Aust Fam Physician 2010; 39:468-474.

7. Nazarian LN. The top 10 reasons musculoskeletal sonography is an important complementary or alternative technique to MRI. AJR Am Roentgenol 2008; 190:1621-1626. 